\title{
Behavior of Vortex-Induced Vibration of A Circular Cylinder Near A Deformable Wall with Two Degrees of Freedom in Steady Flow ${ }^{*}$
}

\author{
YANG Bing (杨 兵) $)^{\mathrm{a}, 1}$, MA Jian-lin (马建林) $)^{\mathrm{a}}$, CUI Jin-sheng (崔金声) ${ }^{\mathrm{b}}$ \\ and XU Wan-hai (徐万海) ${ }^{\mathrm{c}}$ \\ ${ }^{a}$ School of Civil Engineering, Southwest Jiaotong University, Chengdu 610031, China \\ ${ }^{\mathrm{b}}$ Institute of Mechanics, Chinese Academy of Sciences, Beijing 100190, China \\ ${ }^{\mathrm{C}}$ School of Civil Engineering, Tianjin University, Tianjin 300072, China
}

(Received 8 March 2011; received revised form 1 June 2011; accepted 20 July 2011)

\begin{abstract}
The behavior of vortex-induced vibration of a two-degree-of-freedom cylinder near a deformable wall in steady flow is investigated experimentally. The typical phenomenon of the two-degree-of-freedom cylinder's VIV is discussed. The influences of initial gap between the cylinder and the wall on the dynamic responses of the cylinder are analyzed. The comparison is made about dynamic responses of the cylinder with one and two degrees of freedom. Experimental results show that the vibration of the cylinder near a deformable wall with a small value of initial gap-to-diameter ratios can generally be divided into two phases. The initial gap-to-diameter ratios have a noticeable influence on the occurrence of transverse vibration. The transverse maximum amplitude of the cylinder with two degrees of freedom is larger than that of the cylinder with one degree of freedom under the condition with the same values of other parameters. However, the vibration frequency of the cylinder for the two degrees of freedom case is smaller than that for the one degree of freedom case at the same value of Vr number.
\end{abstract}

Key words: vortex-induced vibration; two degrees of freedom; deformable wall; circular cylinder; steady flow

\section{Introduction}

The vortex-induced vibration (VIV) of structures is always a research hotspot due to its practical background and scientific value. The structures in many industries, which, for example, are high chimneys in chemical engineering, high voltage cable, long-span bridges, suspended submarine pipeline, and so on, may suffer vortex-induced vibration. The strong coupling between fluid and structures and high nonlinearity of its governing equation lead to its complexity and difficulties to understand it, which drives many researchers to investigate the mechanism of interaction between fluid and structures. Among the structures, the cylinder is the main object investigated by many researchers, because it has a good representation for many structures.

So far, there exist many studies on the VIV of cylinders, among which the transverse VIV (one degree of freedom), the VIV of the cylinder with two degrees of freedom for the wall-free case, and the VIV of the cylinder with one or two degrees of freedom for the near-wall case are concerned.

Most existing studies have focused on the transverse VIV of the cylinder with one degree of

* This work was financially supported by the National Natural Science Foundation of China (Grant No. 10902112).

1 Corresponding author. E-mail: yangb@swjtu.edu.cn 
freedom due to the larger amplitude in the transverse direction than that in the streamwise direction. The typical works at the early stage are those by Feng (1968) and Anand (1985). They investigated the characteristics of the cylinder's vibration amplitude and frequency in air flow and water flow, respectively. They demonstrated that the vortex shedding frequency locks into the vibration frequency of the cylinder at the lock-in range. In the work by Feng (1968), the vibration frequency of the cylinder in the air flow is nearly a constant and consistent with the natural frequency of the cylinder, but in the results by Anand (1985) the vibration frequency of the cylinder in the water flow increases monotonously with reduced velocity at the lock-in range. According to the results by Govardhan and Williamson (2000), the difference of vibration frequency response between those by Feng (1968) and Anand (1985) can attribute to the larger difference of the mass ratio. The mass ratio of the cylinder used by Feng (1968) is of the order 100, but that by Anand (1985) is 10 or less.

Based on the work by Feng (1968) and Anand (1985), Brika and Laneville (1993) discussed the hysteresis loop of vibration amplitude, vortex shedding mode and the phase difference between the cylinder displacement and the vortex shedding under the condition of high mass ratio. Later, Khalak and Williamson (1996, 1997a and b, 1999) and Govardhan and Williamson (2000) investigated the dynamic response of the cylinder with low mass ratios and low mass-damping parameters. They found that there exist three branches for the amplitude response, i.e. initial branch, upper branch and lower branch. The transition between the initial and upper branches is hysteretic, while it is intermittent between the upper and lower branches. It was confirmed by Govardhan and Williamson (2000) that the initial and lower branches correspond to the $2 \mathrm{~S}$ and $2 \mathrm{P}$ vortex shedding mode, respectively. Also, a concept of critical mass and an expression of vibration frequency versus mass ratio for the lower branch were provided by Govardhan and Williamson (2000, 2004), who (2006) studied the effect of Reynolds number on the amplitude response by controlling damping, and found that the peak-amplitude data collapse well if the effect of Reynolds number is taken into account as an extra parameter in a modified Griffin plot. Raghavan and Bernitsas (2011) also investigated the effect of Reynolds number on vortex-induced vibration. Recently, some works about the VIV are studied further (Horowitz and Williamson, 2010; Farshidianfar and Zanganeh, 2010; Tang et al., 2011; Chen et al., 2011).

As for the vortex-induced vibration of the wall-free cylinder with two degrees of freedom, there is not much research concerning it (Williamson and Govardhan, 2004; 2008). Moe and Wu (1990) and Sarpkaya (1995) found that the position of the maximum response amplitude of the cylinder with two degrees of freedom shifts to a higher value of reduced velocity and the maximum amplitude also reaches a little higher value under the condition of different mass ratios and natural frequencies in the streamwise and transverse direction, as compared with those of transverse-motion ones. Jauvtis and Williamson (2003) studied the response of an elastically mounted cylinder with two degrees of freedom with low mass-damping parameter. They found that the freedom to oscillate in-line with the flow affects the transverse vibration very slightly for $m^{*} \geq 5$ (where $m^{*}$ is the ratio of oscillating structure mass and displaced fluid mass). The experimental results by Williamson and Jauvtis (2004) indicated that there is much difference between the response of the cylinder with one degree of freedom and that with two degrees of freedom for the case of $m^{*}<6$. Blevins and Coughran (2009) found that two degrees-of-freedom motion has a larger velocity entrainment band than transverse-only motion. Huang 
et al. (2011) indicated that the ratio of the frequencies and amplitudes of in-line and cross flow VIV of the cylinders changes with Reynolds number.

In fact, under some circumstances the cylinder will be close to a wall (e.g. the pipelines installed on the seabed). Jacobsen et al. (1984) studied the amplitude response of a cylinder suspended in a spring system near a rigid wall with gap ratios equal to $0,0.5$ and 1 . Based on the work by Jacobsen et al. (1984), Fredsoe et al. (1985) investigated the cross-flow vibration of cylinders near a rigid wall, and their study indicated that the transverse vibration frequency is close to the frequency of vortex shedding from a stationary cylinder, when reduced velocity is less than the value of 3 and the initial gap between the cylinder and the wall $\left(e_{0}\right)$ is more than $0.3 D$. For the case of $3<\mathrm{Vr}<8$ and $0<e_{0}<1 D$ the transverse vibrating frequency is noticeably larger than the frequency of vortex shedding from a stationary cylinder. Raven and Stuart (1985) carried out the full-scale tests on the vortex-induced vibration of the pipeline, and it indicated that the critical Vr number for the occurrence of vibration can be affected by the initial gap. Yang et al. (2006) discussed the transverse VIV of the cylinder near a rigid wall in unidirectional currents and mainly analyzed the dynamic response of the cylinder for the case of $e_{0} / D<0.3$.

Wilson and Caldwell (1971) and King and Jones (1980) preliminarily studied the effect of the proximity to a plane boundary on the vortex-induced vibration of flexible cylinders exposed to steady flow experimentally. Tsahalis and Jones (1981) investigated the VIV of a flexible cylinder with $e_{0} / D=$ $1.0 \sim 6.0$ in steady currents, and found that the maximum amplitude occurred at a higher value of reduced velocity and the maximum amplitude is reduced due to the proximity to the boundary. However, the results by Wilson and Caldwell (1971), King and Jones (1980) and Tsahalis and Jones (1981) did not present the vibration characteristics of the cylinder in streamwise direction, respectively. Later, Tsahalis (1984) studied the vibration amplitude and frequency of a flexible cylinder with $e_{0} / D=1$ and $\infty$ in streamwise and transverse directions under the action of steady currents respectively, and found that the proximity to the plane boundary has a pronounced effect on the amplitude response in both directions. Based on the previous work by Tsahalis (1984), Yang et al. (2008a) investigated the typical characteristics of the transverse and streamwise vibrations of a cylinder with the proximity to the rigid wall extensively through description of vibration amplitude and measurement of vortex shedding frequency at the wake flow region of the cylinder.

In the aforementioned studies, the cylinders are of wall-free conditions or close to rigid walls. In some environments the cylinders may be encountered the deformable walls (e.g. the pipe installed on an erodible sandy bed). Sumer et al. (1988) investigated experimentally the transverse vibration of a circular pipe close to an erodible sandy bed and found that the VIV of the pipe is ultimately dominated by vortex shedding due to the deformability of the wall. On the basis of the work by Sumer et al. (1988), Yang et al. (2008b) further studied the typical characteristics of the transverse vibration of the cylinder near a deformable wall experimentally and discussed the influences of the initial gap between the cylinder and the wall on the cylinder vibration. Shen et al. (2000) made some tentative investigations on the dynamic responses of a cylinder with two degrees of freedom.

It is known from the aforementioned summarization that many useful results have been obtained for the case of wall-free or close to rigid walls. The dynamic responses of the cylinder close to deformable walls are concerned by few researchers. The behavior and mechanism of VIV of the cylinder near a 
deformable wall need to be investigated further.

In this paper, the behavior of vortex-induced vibration of a two-degree-of-freedom cylinder near a deformable wall in steady flow is investigated experimentally. The typical phenomenon of the two-degree-of-freedom cylinder's VIV is discussed extensively. The influences of initial gap between the cylinder and the wall on the dynamic responses of the cylinder are analyzed.

\section{Experimental Set-up}

A special hydro-elastic apparatus is used for the present experiments (as shown in Fig. 1), which is installed in a flume. The flume is $0.5 \mathrm{~m}$ wide, $0.6 \mathrm{~m}$ high and $19 \mathrm{~m}$ long, which can produce steady flow with velocity up to $0.6 \mathrm{~m} / \mathrm{s}$. The water depth is kept at $0.4 \mathrm{~m}$. The experimental cylinder with a diameter of $0.032 \mathrm{~m}$ is attached to the connecting poles which are hinged with sliding pole by a gemel. The sliding pole can only move in the vertical direction due to the restriction of four limit wheels. The movable parts include the cylinder, connecting poles, gemels and sliding poles, which are connected to the supporting frame by a set of vertical springs. Therefore, $y$-component of the test cylinder's mass comprises the masses of the cylinder itself, connecting poles, gemels, sliding poles, and the natural frequency of the cylinder in $y$ direction is determined by $y$-component of the test cylinder's mass and the coefficient of vertical springs. $x$-component of the test cylinder's mass includes only masses of the cylinder itself and part of connecting poles (about 50\% of the mass of connecting poles). In order to model the elasticity of the cylinder in $x$ direction, the horizontal springs are installed at the two ends of the cylinder. In order to reduce the disturbance between the motion of the cylinder in $x$ and $y$ directions, i.e. the motion in two directions are decomposed to the highest extent, the length of the connecting pole (i.e. the distance between gemel and the center of the cylinder) is set to be $0.6 \mathrm{~m}$, and the length of the horizontal spring (i.e. the distance between the center of the cylinder and the fixed point) in still water is set to be $1 \mathrm{~m}$. Thus, the tilt angle for the connecting pole and horizontal spring will be small (about less than $5^{\circ}$ ) during the course of cylinder's vibration. The length of the cylinder is $0.47 \mathrm{~m}$, and the surface of the cylinder is smooth, i.e. $\kappa \approx 0$.

The laser displacement transducers are used to measure the vibration displacements of the cylinder. The one with a dynamic resolution of $0.25 \mathrm{~mm}$ is used for the measurement of the vertical displacement and the one with $0.025 \mathrm{~mm}$ is for the horizontal displacement. The sampling rate of the vibration displacements is set to be $500 \mathrm{~Hz}$. The natural frequency of the cylinder $\left(f_{\mathrm{n}}\right)$ is obtained by spectrum analyses of free-decay tests in still water, and the structural damping of the cylinder is measured with the method of free-decay tests, i.e. the cylinder is given a prescribed displacement and then released in still water. The structural damping factor $(\zeta)$ can be estimated with $\zeta=\ln \left(A_{i} / A_{i+n}\right) /(2 \pi n)$, where $A_{i}$ is the initial amplitude of cylinder vibrations, and $A_{i+n}$ is the amplitude after $n$ cycles (Blevins, 1977). In this study, the value of $n$ is specified as 5 .

A micro-propeller current-meter with a resolution of $0.1 \mathrm{~cm} / \mathrm{s}$ is adopted to measure the coming flow velocity. The micro-propeller current-meter is installed at the depth of $2 / 3 H$ ( $H$ is water depth). Sandy bed with a depth of $15 \mathrm{~cm}$ is used to simulate the deformable wall. The mean grain diameter $\left(d_{50}\right)$ 
is $0.38 \mathrm{~mm}$ and the relative density $\left(D_{\mathrm{r}}\right)$ is 0.66 , indicating that the soil is a medium-dense one.

The gap between the cylinder and the wall (sandy bed) is measured by the dividing ruler installed on the side wall of the flume and the camera. The resolution of the measurement is $0.5 \mathrm{~mm}$.

In every group of experiments, the flow velocity is adjusted gradually when other parameters (e.g. the diameter of the cylinder and the natural frequency of the cylinder) are fixed. Thus, at every flow velocity, the vibration amplitude, vibration frequency of the cylinder and the flow field around the cylinder can be obtained, and each test does not stop until the vibration of the cylinder and the shape of the wall reaches the steady statement.

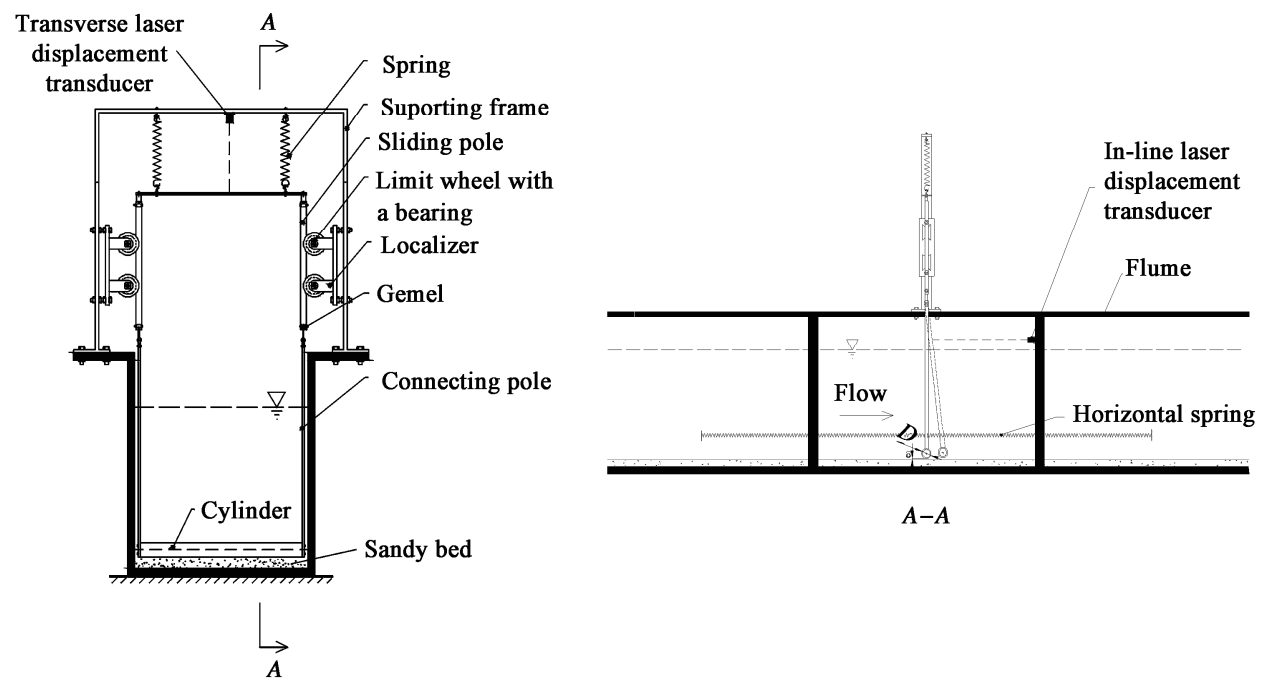

Fig. 1. Schematic diagram of the experimental set-up.

\section{Measurement of Vibration Displacement}

In this study, the measurement of vibration displacement of the cylinder is not easy, because the cylinder was placed in the water. The laser displacement transducer can not work when it suffers the water. In order to obtain the vibration displacement of the cylinder, the displacement of certain point on the apparatus over the surface of water is measured. The displacement of the cylinder can be calculated by the geometrical relationship between the point measured and the center of the cylinder. Fig. 2 presents the principle of measurement. In the figure the origin of coordinate is set at the center of static cylinder, and the coordinate axes of $x$ and $y$ are along the horizontal and vertical directions, respectively. Point $B$ represents the center of the cylinder. It can be seen from Fig. 2 that the vibration displacement of the cylinder will be known if the coordinate of point $B^{\prime}$ is known. According to the geometrical relationship in the figure, the relation between the coordinate of point $A^{\prime}$ and that of $B^{\prime}$ can be written as:

$$
x=\frac{a+b}{a} x_{1}
$$




$$
y=y_{1}-b \frac{\sqrt{a^{2}-x_{1}^{2}}}{a} .
$$

in which $x$ and $y$ are the coordinates of point $B^{\prime}, x_{1}$ and $y_{1}$ are those of point $A^{\prime} . a$ is the distance between the swing joint of the connecting pole and the point on which the horizontal laser beam acts at the connecting pole when the cylinder is at the initial place, and equals about $0.1 \mathrm{~m} . b$ is the distance between the horizontal laser beam acting point at the connecting pole and the center of the cylinder for the initial state, and equals about $0.5 \mathrm{~m}$. Based on the geometry deduction, the coordinates of point $A^{\prime}$ can be written as:

$$
\begin{aligned}
& x_{1}=\left(x^{\prime}-\Delta y \frac{x^{\prime}}{a+\Delta y}\right) \sqrt{\frac{1}{1+\left[\left(x^{\prime}-\Delta y \frac{x^{\prime}}{a+\Delta y}\right) / a\right]^{2}}} ; \\
& y_{1}=b+\Delta y+a\left\{1-\sqrt{\frac{1}{1+\left[\left(x^{\prime}-\Delta y \frac{x^{\prime}}{a+\Delta y}\right) / a\right]^{2}}}\right\} .
\end{aligned}
$$

where $x^{\prime}$ is the horizontal displacement of the connecting pole in the laser beam direction, $\Delta y$ is the displacement of point $A$ in the vertical direction. The displacement ( $\left.x^{\prime}\right)$ can be measured directly by the horizontal laser displacement transducer, and the displacement $(\Delta y)$ equals approximately the value measured by the vertical laser displacement transducer due to the small swing angle of the connecting pole (less than $5^{\circ}$, the error is about $x^{\prime} \tan \phi$, and $\phi$ is the tilt angle of the connecting pole). Therefore, the vibration displacement of the cylinder can be obtained from Eqs. (1) (4).

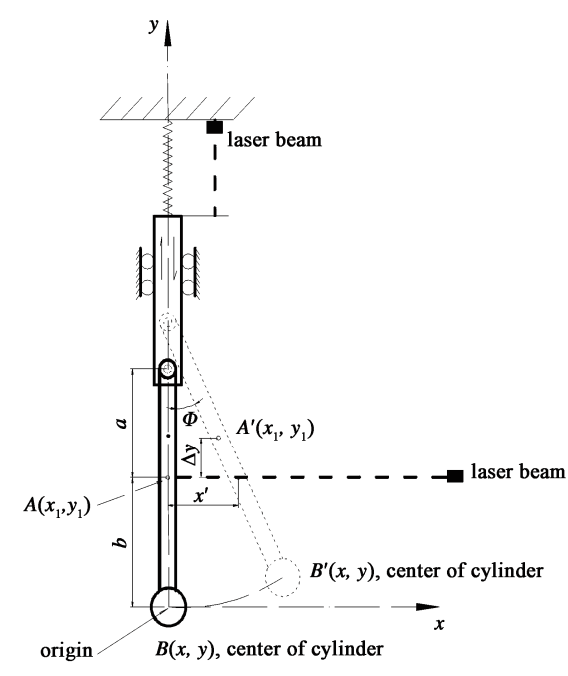

Fig. 2. Schematic diagram for the measurement of the cylinder's vibration displacement $(b=4 a)$. 
According to Eq. (3), the resolution of the horizontal coordinate $x_{1}$ is related to the resolution of $x^{\prime}$ and $\Delta y$. As the maximum of the cylinder's horizontal displacement is not more than $20 \mathrm{~mm}$ which is known from the results in this test, the tilt angle of the connecting pole is less than $2^{\circ}$. The resolution of $x^{\prime}$ is $0.025 \mathrm{~mm}$. Therefore, the resolution of $\Delta y$ is about $x^{\prime} \tan \phi+0.25 \mathrm{~mm}$, i.e. $0.37 \mathrm{~mm}$. It is indicated from Eqs. (3) and (4) that the resolution of $x_{1}$ is about $0.03 \mathrm{~mm}$, and that of $y_{1}$ is about $0.39 \mathrm{~mm}$. According to Eqs. (1) and (2), the resolution of $x$ and $y$ can be known, i.e. $0.18 \mathrm{~mm}$ for $x$ coordinate and $0.39 \mathrm{~mm}$ for $y$ coordinate. So the resolution of the streamwise vibration displacement measured in the experiments is $0.18 \mathrm{~mm}$, and the resolution of the transverse one is $0.39 \mathrm{~mm}$.

\section{Results and Discussion}

\subsection{Dimensionless Parameters}

The dynamic response of the cylinder near a deformable wall in steady flow involves the characteristics of fluid, cylinder and deformable wall. The physical quantities influencing the dynamic response of the cylinder are described in Table 1.

Table 1 Physical quantities related to dynamic response of the cylinder near a deformable wall in steady flow

\begin{tabular}{|c|c|c|c|c|c|}
\hline Physical quantities & Symbols & Dimensions & Physical quantities & Symbols & Dimensions \\
\hline \multicolumn{3}{|c|}{ Relative to fluid } & \multicolumn{3}{|c|}{ Relative to the cylinder } \\
\hline Mass density of fluid & $\rho$ & $\mathrm{ML}^{-3}$ & Diameter of the cylinder & $D$ & $\mathrm{~L}$ \\
\hline Dynamic viscosity of fluid & $\mu$ & $\mathrm{ML}^{-1} \mathrm{~T}^{-1}$ & $\begin{array}{l}\text { Relative roughness of the cylinder's } \\
\text { surface }\end{array}$ & $\kappa$ & 1 \\
\hline \multirow[t]{3}{*}{$\begin{array}{l}\text { Undisturbed incoming flow } \\
\text { velocity }\end{array}$} & $U$ & $\mathrm{LT}^{-1}$ & Mass of the cylinder per meter & $m$ & M \\
\hline & & & $\begin{array}{l}\text { Natural frequency of the cylinder in still } \\
\text { water }\end{array}$ & $f_{\mathrm{n}}$ & $\mathrm{T}^{-1}$ \\
\hline & & & Structural damping factor of the cylinder & $\zeta$ & 1 \\
\hline \multicolumn{3}{|c|}{ Relative to the sand } & \multicolumn{3}{|l|}{ Other quantities } \\
\hline Mass density of sand grain & $\rho_{\mathrm{s}}$ & $\mathrm{ML}^{-3}$ & Duration of loading & $t$ & $\mathrm{~T}$ \\
\hline Mean diameter of sand grain & $d_{50}$ & $\mathrm{~L}$ & Gravitational acceleration & $g$ & $\mathrm{LT}^{-2}$ \\
\hline Relative density of sandy soil & $D_{\mathrm{r}}$ & 1 & $\begin{array}{l}\text { Initial gap between cylinder and plane } \\
\text { boundary }\end{array}$ & $e_{0}$ & $\mathrm{~L}$ \\
\hline
\end{tabular}

According to PI theorem, the physical quantities in Table 1 can constitute the following dimensionless parameters (see Table 2)

Therefore, the vibration amplitude $(A)$ and frequency $(f)$ can be expressed as:

$$
\begin{aligned}
& \frac{A}{D}=\varphi\left[m^{*}, V_{\mathrm{r}}, \kappa, K_{\mathrm{s}}, \operatorname{Re}, G_{\mathrm{s}}, d_{50} / D, \theta, D_{\mathrm{r}}, e_{0} / D,(t U) / D\right] ; \\
& \frac{f}{f_{n}}=\varphi\left[m^{*}, V_{\mathrm{r}}, \kappa, K_{\mathrm{s}}, \operatorname{Re}, G_{\mathrm{s}}, d_{50} / D, \theta, D_{\mathrm{r}}, e_{0} / D,(t U) / D\right] .
\end{aligned}
$$

In this paper the parameters, i.e. $\kappa, G_{\mathrm{s}}, d_{50} / D$ and $D_{\mathrm{r}}$, are fixed for all tests, and $\kappa \approx 0, G_{\mathrm{s}}=2.65$, 
$d_{50} / D=0.0119$, and $D_{\mathrm{r}}=0.66$. The parameters (i.e. $m^{*}$ and $K_{\mathrm{s}}$ ) are fixed in each group of test.

Table 2 Dimensionless parameters related to dynamic response of the cylinder near a deformable wall in steady flow

\begin{tabular}{|c|c|c|c|c|c|}
\hline $\begin{array}{c}\text { Dimensionless } \\
\text { parameters }\end{array}$ & Symbols & Definitions & Dimensionless parameters & Symbols & Definitions \\
\hline Mass ratio & $m^{*}$ & $(4 m) /\left(\pi \rho D^{2}\right)$ & $\begin{array}{l}\text { Dimensionless diameter of } \\
\text { sand grain }\end{array}$ & $d_{50} / D$ & \\
\hline Reduced velocity & $V_{\mathrm{r}}$ & $U /\left(f_{\mathrm{n}} D\right)$ & Shields number & $\theta$ & $u_{f} / \sqrt{\left(G_{\mathrm{s}}-1\right) g d_{50}}$ \\
\hline Relative roughness & $\kappa$ & & Relative density of sandy soil & $D_{\mathrm{r}}$ & \\
\hline Stability parameter & $K_{\mathrm{S}}$ & {$\left[4\left(m+m_{\mathrm{a}}\right) \zeta\right] /\left(\pi \rho D^{2}\right)$} & Gap-to-diameter ratio & $e_{0} / D$ & \\
\hline Reynolds number & $\operatorname{Re}$ & $(U D) / v$ & Dimensionless loading time & $(t U) / D$ & \\
\hline $\begin{array}{l}\text { Specific gravity of sand } \\
\text { grain }\end{array}$ & $G_{\mathrm{s}}$ & $\rho_{\mathrm{s}} / \rho$ & & & \\
\hline
\end{tabular}

Note: In Table $2, m_{\mathrm{a}}$ is the added mass and $m_{\mathrm{a}}=C_{\mathrm{A}} \cdot m_{\mathrm{d}}, C_{\mathrm{A}}$ is the coefficient of added mass, $C_{\mathrm{A}} \approx 1.0$ and $m_{\mathrm{d}}=\left(\pi \rho D^{2}\right) / 4$ for a cylinder. $u_{f}$ is the bed shear velocity and can be calculated with the Colebrook-White formula, i.e. $U / u_{f}=8.6+2.5 \ln \left(D / 2 k_{\mathrm{b}}\right)$, in which $k_{\mathrm{b}}$ is the roughness of bed, and is usually taken as $2.5 d_{50}$ (Sumer and Fredsoe, 2002).

\subsection{Typical Phenomenon of the Cylinder's Vibration}

Fig. 3 shows the typical characteristics of scour and the vibration displacement of the cylinder for the case of $e_{0} / D=-0.25$ (the cylinder is embedded in the sandy bed). The experimental parameters in the figure are as follows: $m_{x}{ }^{*}=1.97, \zeta_{x}=0.0275\left(K_{\mathrm{s}}=0.0817\right), m_{y}{ }^{*}=2.60, \zeta_{y}=0.0391 \quad\left(K_{\mathrm{s}}=0.1408\right)$, $f_{\mathrm{nx}} / f_{\mathrm{n} y}=1, f_{\mathrm{nx}}=1.00 \mathrm{~Hz}, U=0.255 \mathrm{~m} / \mathrm{s}, \mathrm{Re}=8160, \theta=0.039$. It is indicated from Figs. 3(a) and (b) that the vibration of the cylinder can be divided into two phases. In the first phase the cylinder is static due to the small gap between the cylinder and sandy bed, and the vortexes in the wake of the cylinder are suppressed by the wall. However, the boundary deforms continuously under the action of fluid, namely, the gap between the cylinder and sandy bed (wall) increases steadily (see Fig. 3(c)). When the gap between the cylinder and sandy bed increases up to $20.5 \mathrm{~mm}$, i.e. $e_{t} / D=0.64$ (see Fig.3(c)), the cylinder begins to vibrate. In this case the influence of the wall on the vortex shedding weakens. The fluctuating lift and drag forces are against the damping force of the cylinder, so the vibration displacement of the cylinder can be observed. Subsequently, the vibration enters into the second phase. At the initial time of this phase, since the cylinder impacts the sandy bed and the scour develops sharply, the gap between the cylinder and sandy bed increases rapidly. It is shown from Fig. 3(c) that the value of gap changes from $20.5 \mathrm{~mm}$ to $30 \mathrm{~mm}$ within the time about $1800 \mathrm{~s}$ in the second phase, and the speed of variation is obviously larger than that in the first phase. When the gap gets up to $30 \mathrm{~mm}$, i.e. $e_{t} / D=0.94$, the fluid force acting on the sandy bed decreases, and the gap between the cylinder and sandy bed varies little. In this case scour gets into the stage of equilibrium scour. After the occurrence of vibration, the vibration amplitudes in streamwise and transverse directions vary steadily, but there exist multiple components of vibration frequency during the course of cylinder's vibration in two directions (Figs. 3(a) and (b)). In order to understand the phenomenon of multiple components, the power spectrums of vibration displacement at different time are plotted in Fig. 4 (taking streamwise vibration 
as example). It can be seen from Fig. 4 that the streamwise vibration frequency of the cylinder decreases gradually with the time, namely, the vibration frequency will be influenced by the gap ratio. As the gap ratio will increase with the time, the vibration frequency will become larger with the increase of gap ratio. On the other hand, the vortex shedding frequency and natural frequency of the cylinder interact each other, thus their several coupling frequencies appear. Therefore, there exist different peak frequencies from initial to equilibrium stages. Thus the multiple components of frequency will appear in the power spectrum of the streamwise vibration curves for the whole time (from $3800 \mathrm{~s}$ to $5950 \mathrm{~s}$ ). According to the results by Fredsoe et al. (1985) and Yang et al. (2006), the gap between the cylinder and the wall will affect the vibration frequency of the cylinder, and the vibration frequency tends to decrease with the increasing gap. In the present study the gap between the cylinder and the wall continues to increase during the vibration due to the deformability of the wall, so the vibration frequency of the cylinder shows a decline trend, which is consistent with the results by Fredsoe et al. (1985) and Yang et al. (2006). Through the same analysis, the aforementioned conclusions also apply for the case of transverse vibration of the cylinder.

\section{Influence of Gap-to-diameter Ratio on the Dynamic Response of the Cylinder}

In this section the characteristics of vortex-induced vibration of the cylinder with two degrees of freedom for the case of various gap-to-diameter ratios will be analyzed. Fig. 5 illustrates the amplitude response of the cylinder. It is indicated from the figure that when the initial gap-to-diameter ratios are smaller (e.g. $e_{0} / D=0.34,0,-0.25$ ), and the critical Vr number, at which the transverse vibration of the cylinder begins to appear, becomes higher (e.g. $\mathrm{Vr}_{\mathrm{ctr}} \approx 7.8$ ). On the other hand, when the initial gap-to-diameter ratios are larger (e.g. $e_{0} / D=0.56,0.94,1.47,2.72$ ), the critical Vr number becomes lower (e.g. $\mathrm{Vr}_{\mathrm{ctr}} \approx 3.4$ ). The main cause is that the scour around the cylinder will occur at larger velocity. The scour around the cylinder will lead to the increase of gap between the cylinder and the sandy bed, so the vortex shedding in the wake flow of the cylinder will be strengthened due to the change of gap. It also can be seen from the figure that the first streamwise vibration does not exist for the case of smaller gap-to-diameter ratios due to the suppression of vortex shedding, and it appears for the case of larger ones. The above conclusions are consistent with those for the case of rigid wall (Yang et al., 2008a).

Fig. 6 shows the frequency response of the cylinder with various initial gap-to-diameter ratios at the equilibrium stage. As can be seen from the figure, the initial gap-to-diameter ratios affect the vibration frequency of the cylinder slightly. For the smaller gap-to-diameter ratios (see Fig. $6\left(a_{1}\right)$ and $6\left(b_{1}\right)$ ) the dimensionless vibration frequencies of the cylinder with various initial gap-to-diameter ratios nearly vary along the same curve with the increase of Vr number. For the case of larger ones, the vibration frequency tends to increase at the same value of $\mathrm{Vr}$ number with the increase of gap ratio, but growth rate is very small (see Fig. $6\left(a_{2}\right)$ and $6\left(b_{2}\right)$ ). The possible reasons for the results above are that when the scour develops fully and stays at the equilibrium stage, the vortex shedding in the wake flow is nearly not affected by the wall (sandy bed) in this case. Therefore, the characteristics of the cylinder with various gap-to-diameter ratios are similar at the equilibrium stage. 

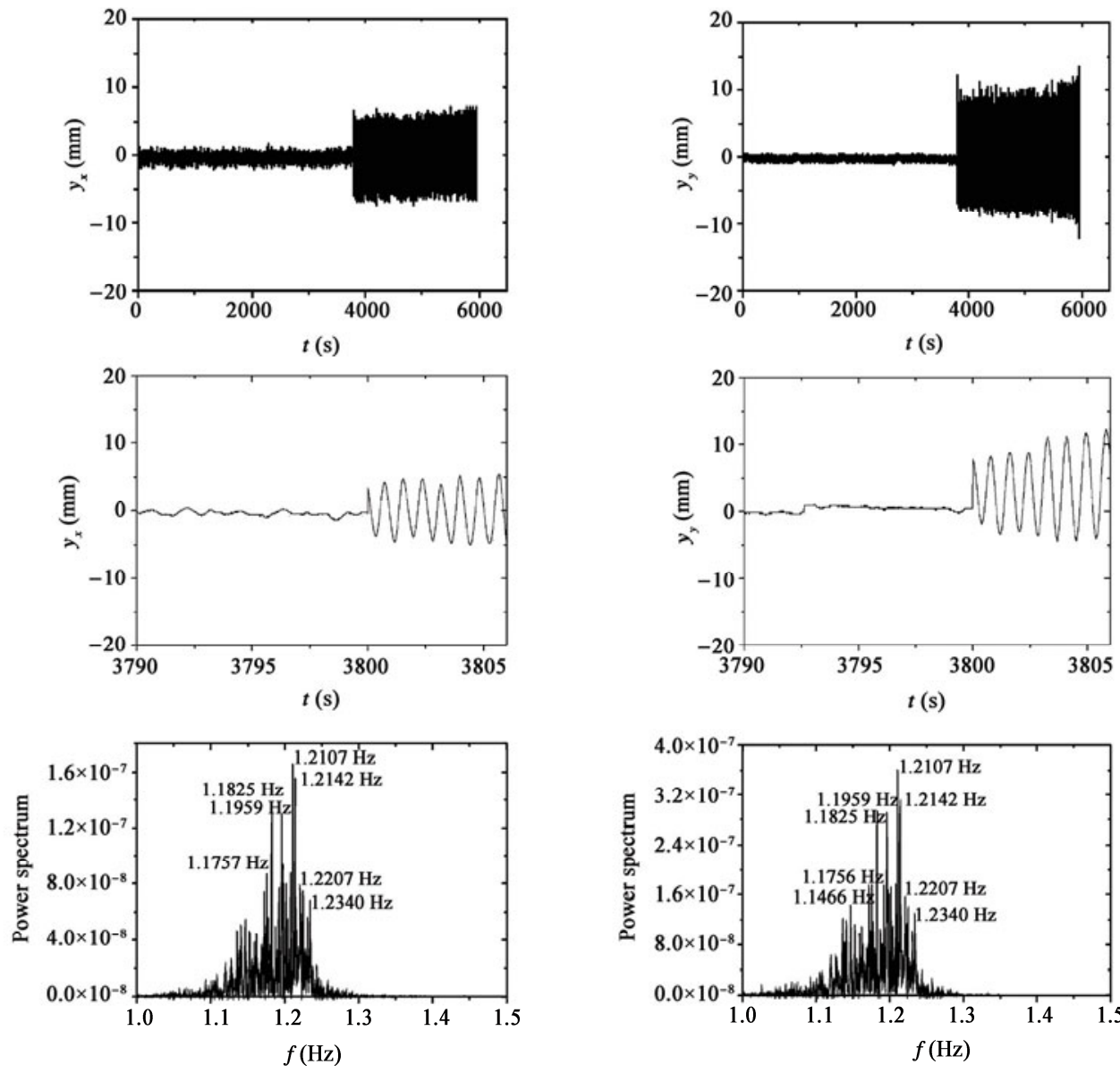

(a) Streamwise vibration displacement and corresponding power spectrum

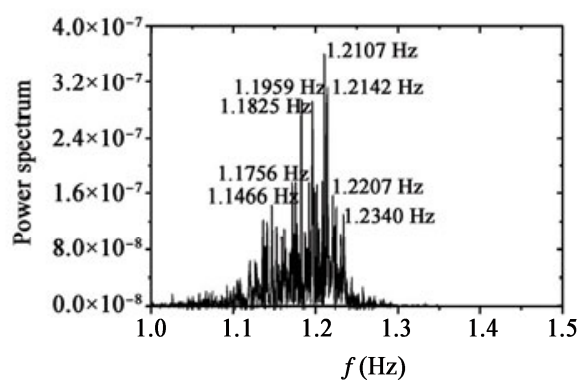

(b) Transverse vibration displacement and corresponding power spectrum

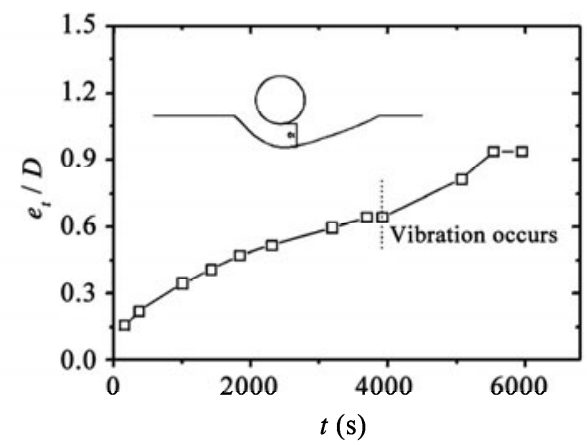

(c) Variation of gap between circular cylinder and sandy bed versus time

Fig. 3. Variation of scour and the cylinder's displacement with time for the case of $e_{0} / D=-0.25\left(D=0.032 \mathrm{~m}, e_{0} / D=-0.25\right.$, $m_{x}^{*}=1.97, \zeta_{x}=0.0275\left(K_{\mathrm{s}_{\mathrm{x}}}=0.0817\right), m_{y}^{*}=2.60, \zeta_{y}=0.0391\left(K_{\mathrm{s}_{\mathrm{y}}}=0.1408\right), f_{\mathrm{n} x}=1 \mathrm{~Hz}, f_{\mathrm{n} x} / f_{\mathrm{n} y}=1, U=0.255 \mathrm{~m} / \mathrm{s}$, $\mathrm{Re}=8160, \theta=0.039$, medium sand). 

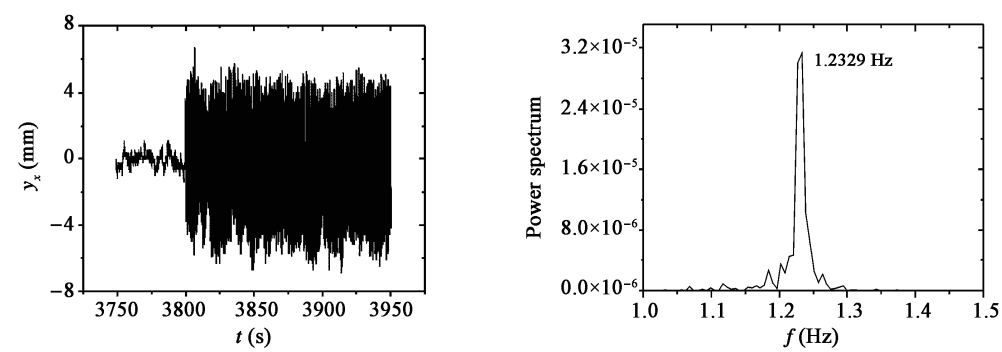

(a) Vibration displacement and corresponding power spectrum at the stage of $3800 \mathrm{~s} \sim 3950 \mathrm{~s}$

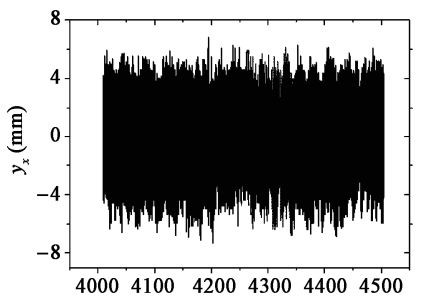

$t(\mathrm{~s})$

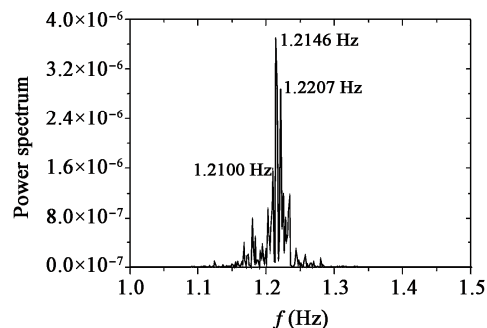

(b) Vibration displacement and corresponding power spectrum at the stage of $4000 \mathrm{~s} \sim 4500 \mathrm{~s}$
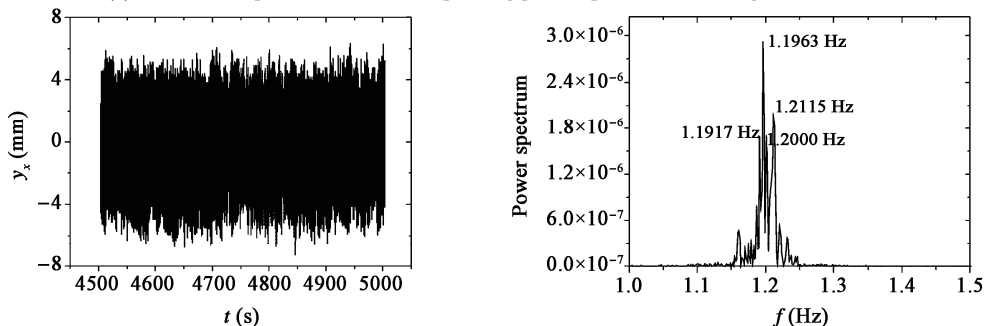

(c) Vibration displacement and corresponding power spectrum at the stage of $4500 \mathrm{~s} \sim 5000 \mathrm{~s}$

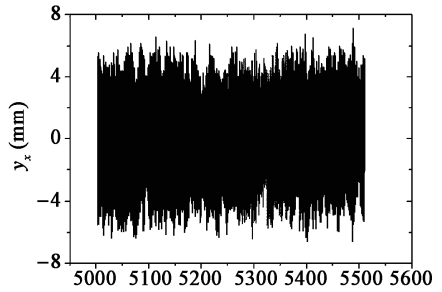

$t(\mathrm{~s})$

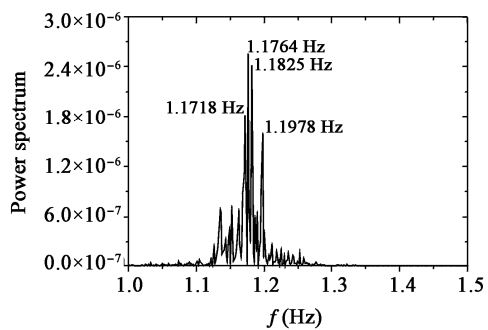

(d) Vibration displacement and corresponding power spectrum at the stage of $5000 \mathrm{~s} \sim 5500 \mathrm{~s}$
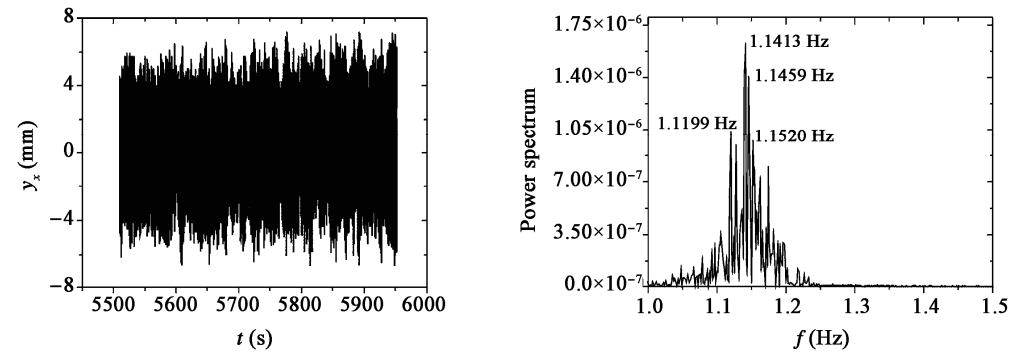

(e) Vibration displacement and corresponding power spectrum at the stage of $5500 \mathrm{~s} \sim 5950 \mathrm{~s}$

Fig. 4. Variation of the cylinder's vibration frequency with time (in streamwise direction). 


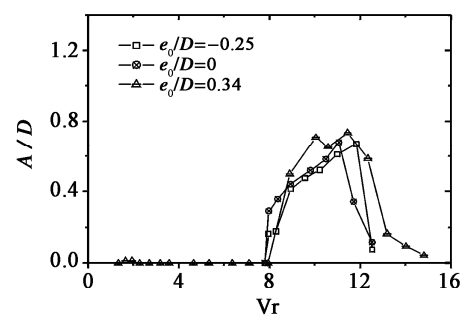

(a) Streamwise vibration amplitude response

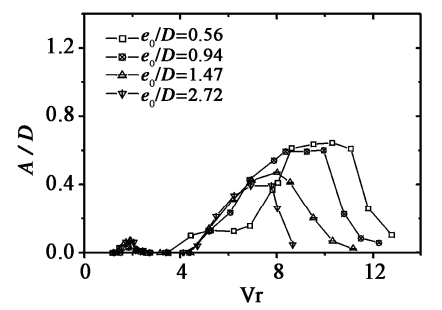

$\left(a_{2}\right)$ Streamwise vibration amplitude response

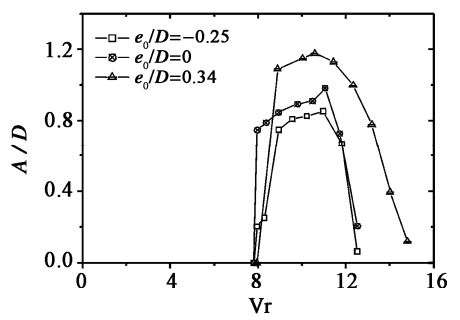

(b) Transverse vibration amplitude response

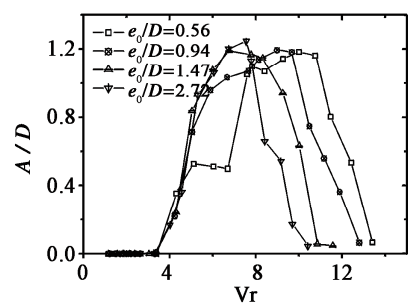

$\left(b_{2}\right)$ Transverse vibration amplitude response

Fig. 5. Amplitude response of vortex-induced vibration of the cylinder with two degrees of freedom for the case of various gap-to-diameter ratios $\left(D=0.032 \mathrm{~m}, m_{x}^{*}=1.97, \zeta_{x}=0.0386\left(K_{\mathrm{s}_{\mathrm{x}}}=0.1146\right), m_{y}^{*}=2.60, \zeta_{y}=0.0569 \quad\left(K_{\mathrm{s}_{\mathrm{y}}}=0.2048\right)\right.$, $f_{\mathrm{n} x} / f_{\mathrm{n} y}=1$, medium sand).

For the case of larger gap ratios (e.g. $e_{0} / D=0.56,0.94,1.47,2.72$ ), in the region of the second streamwise vibration, the streamwise vibration frequency undergoes a jump at certain value of $\mathrm{Vr}$ number, because at the position of jump the streamwise vibration frequency is twice as much as that of transverse one.

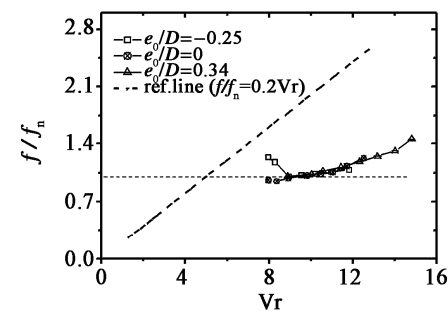

$\left(a_{1}\right)$ Streamwise vibration frequency response

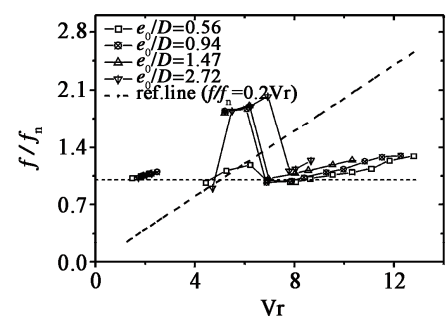

(a) Streamwise vibration frequency response

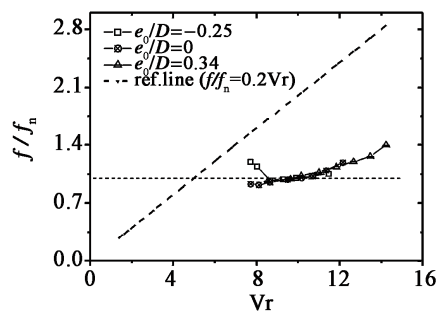

$\left(b_{1}\right)$ Transverse vibration frequency response

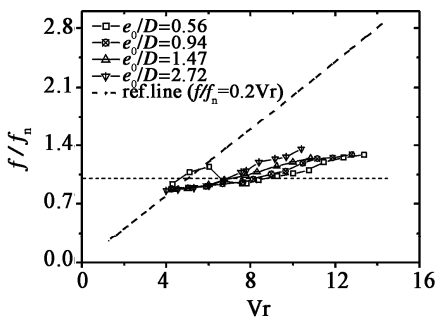

$\left(b_{2}\right)$ Transverse vibration frequency response

Fig. 6. Frequency response of vortex-induced vibration of the cylinder with two degrees of freedom for the case of various gap-to-diameter ratios $\left(D=0.032 \mathrm{~m}, m_{x}^{*}=1.97, \zeta_{x}=0.0386\left(K_{\mathrm{s}_{x}}=0.1146\right), m_{y}^{*}=2.60, \zeta_{y}=0.0569 \quad\left(K_{\mathrm{s}_{y}}=0.2048\right)\right.$, $f_{\mathrm{n} x} / f_{\mathrm{n} y}=1$, medium sand). 


\section{Comparison of Dynamic Response of the Cylinder with One and Two Degrees of Freedom}

In this section the comparison is made on the characteristic of transverse vortex-induced vibration of the cylinder with one and two degrees of freedom, as illustrated in Fig. 7. It can be seen from the figure that the maximum amplitude of the cylinder's transverse vibration with two degrees of freedom is larger than that of the cylinder with one degree of freedom. However, the vibration frequency of the cylinder for the two degrees of freedom case is smaller than that for the one degree of freedom case at the same value of Vr number. For the case of two degrees of freedom, the motion of the cylinder has one more degree of freedom than that of one-degree-of-freedom cylinder, so the cylinder with two degrees of freedom can absorb more energy to support its larger amplitude motion. On the other hand, due to the existence of streamwise motion the speed of motion for two-degree-of-freedom cylinder is slower than that for one-degree-of-freedom cylinder, so the vibration frequency of the cylinder with two degrees of freedom is less than that of one-degree-of-freedom cylinder.

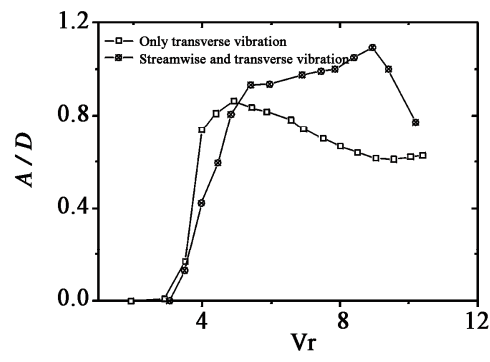

$\left(a_{1}\right)$ Amplitude response

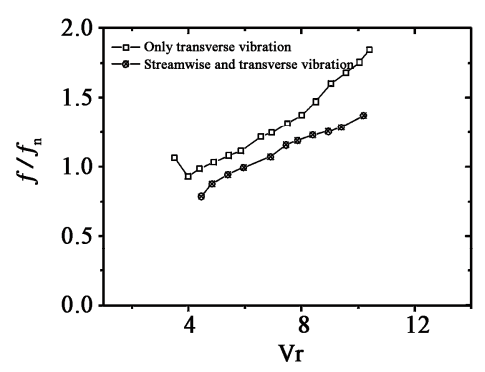

$\left(a_{2}\right)$ Frequency response

(a) $\left(D=0.032 \mathrm{~m}, e_{0} / D=2.00\right.$; one degree of freedom: $m^{*}=1.36, \zeta=0.596\left(K_{s}=0.1407\right)$; two degrees of freedom: $m_{y}^{*}=1.36, \zeta_{y}=0.0596,\left(K_{s_{y}}=0.1407\right), m_{x}^{*}=1.16, \zeta_{x}=0.0352,\left(K_{s_{x}}=0.0760\right) ; f_{\mathrm{nd}}=f_{\mathrm{nx}}=f_{\mathrm{ny}}$, medium sand $)$

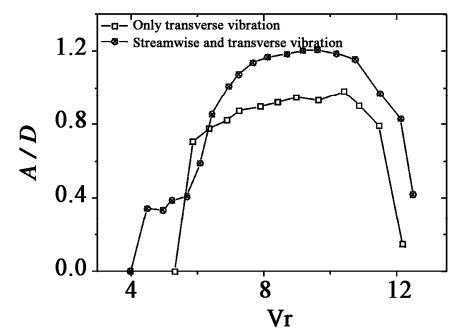

( $\left.a_{1}\right)$ Amplitude response

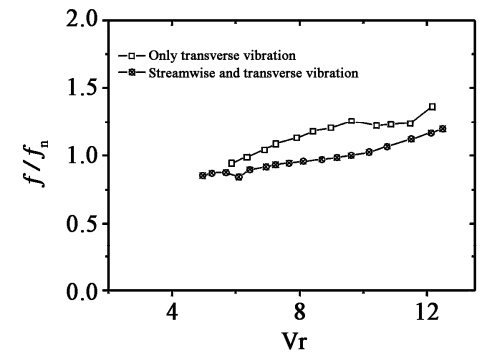

$\left(a_{2}\right)$ Frequency response

(b) $\left(D=0.032 \mathrm{~m}, e_{0} / D=0.32\right.$; one degree of freedom: $m^{*}=2.99, \zeta=0.0762\left(K_{s}=0.3040\right)$; two degrees of freedom: $m_{y}^{*}=2.99, \zeta_{y}=0.0762,\left(K_{s_{y}}=0.3040\right), m_{x}^{*}=2.67, \zeta_{x}=0.0261,\left(K_{s_{x}}=0.0958\right) ; f_{\mathrm{nd}}=f_{\mathrm{nx}}=f_{\mathrm{ny}}$, medium sand $)$

Fig. 7. Comparison of dynamic response of the cylinder with one and two degrees of freedom.

\section{Comparison with Previous Results}

Fig. 8 presents the comparison of the present study with previous results by Jauvtis and Williamson (2003), Yang et al. (2008a), and Tsahalis et al. (1984). In the results for the case of wall-free cylinder by Jauvtis and Williamson (2003), the mass ratio $m^{*}=2.6$, mass-damping parameter $\left(m^{*}+C a\right) \zeta=0.013$. 
The experimental parameters in the study by Yang et al. (2008a) are set as: $m_{x}^{*}=1.97, \zeta_{x}=0.0431$, $m_{y}{ }^{*}=2.60, \zeta_{y}=0.0569, e_{0} / D=2.68$ for rigid wall. The mass ratio $m^{*}=2.8, \zeta=0.0318$, gap-to-diameter ratio $e_{0} / D=1$ and $\infty$ for rigid wall, are used by Tsahalis et al. (1984). The parameters of the present study are $m_{x}{ }^{*}=1.97, \zeta_{x}=0.0386, m_{y}{ }^{*}=2.60, \zeta_{y}=0.0569, f_{\mathrm{nx}} / f_{\mathrm{n} y}=1$ for deformable wall. It is seen from Fig. 8(a) that there exists streamwise vibration in the range of $1<\mathrm{Vr}<3$ for the results by Jauvtis and Williamson (2003), Yang et al. (2008a) and the present study, and it is not clear for those by Tsahalis et al. (1984). The causes may possibly be the low resolution of the instruments which measure the vibration displacement used by Tsahalis et al. (1984), so that the streamwise vibration in the range of $1<\mathrm{Vr}<3$ is not detected by Tsahalis et al. (1984) due to the small amplitude (about $0.06 \mathrm{D}$ of its maximum amplitude). It is indicated from Fig. 8(a) that the maximum amplitude of transverse vibration and vibration range in terms of Vr number in the results by Jauvtis and Williamson (2003) are larger than those by others, which may possibly attribute to the lower mass-damping parameter used by Jauvtis and Williamson (2003). Fig. 8(a) shows that the transverse vibration amplitude is larger than the streamwise one, which is consistent with the results by Jauvtis and Williamson (2003), Yang et al. (2008a), Tsahalis et al. (1984) and the present study. Fig. 8(b) gives the comparison of frequency response. It can be seen from the figure that there exists the phenomenon of jump for streamwise vibration frequency in the results by Yang et al. (2008a), Tsahalis et al. (1984) with $e_{0} / D=\infty$ and the present study. The jump of streamwise vibration frequency does not occur in the results by Tsahalis et al. (1984) for the case of $e_{0} / D=1$ due to the influence of wall. For the transverse vibration frequency it shows the same trend of variation with Vr number, as depicted in Fig. 8(b).

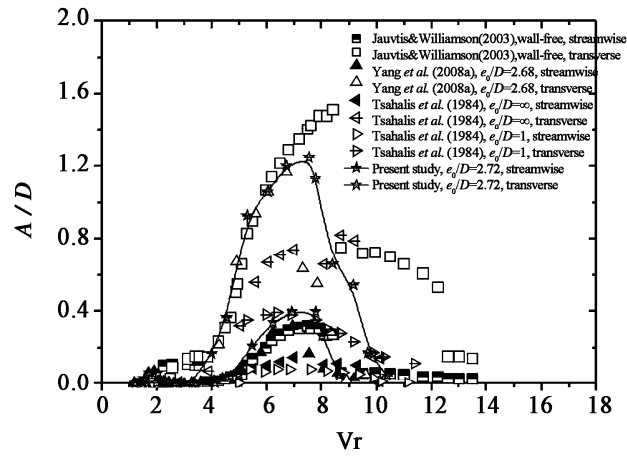

(a) Amplitude response

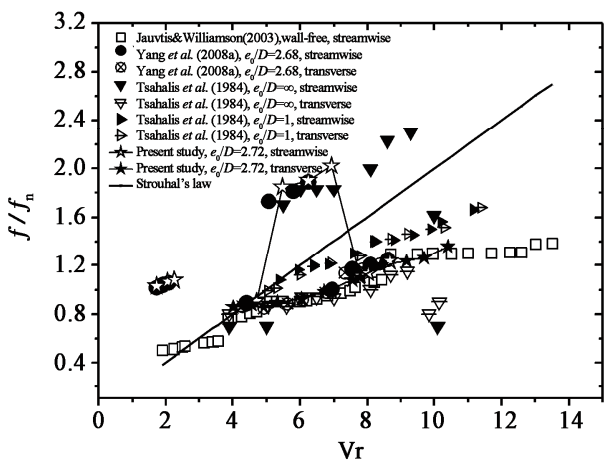

(b) Frequency response

Fig. 8. Comparison with previous results.

\section{Conclusions}

When the initial gap-to-diameter ratios are smaller (e.g. $e_{0} / D=0.34,0,-0.25$ ), the critical $\mathrm{Vr}$ number, at which the transverse vibration of the cylinder begins to appear, becomes higher (e.g. $\mathrm{Vr}_{\mathrm{ctr}} \approx 7.8$ ). The first streamwise vibration does not exist for the case of smaller gap-to-diameter ratios, and it appears for the case of larger ones. 
The initial gap-to-diameter ratios affect the vibration frequency of the cylinder slightly. For the case of larger gap ratios (e.g. $e_{0} / D=0.56,0.94,1.47,2.72$ ), in the region of the second streamwise vibration, the streamwise vibration frequency undergoes a jump at certain value of Vr number.

The transverse vibration maximum amplitude of the cylinder with two degrees of freedom is larger than that of the cylinder with one degree of freedom under the condition of the same values of other parameters. However, the vibration frequency of the cylinder for the two degrees of freedom case is smaller than that for the one degree of freedom case at the same value of Vr number.

\section{References}

Anand, N. M., 1985. Free Span Vibrations of Submarine Pipelines in Steady and Wave Flows, Ph. D. Thesis, The Norwegian Institute of Technology, Trondheim, Norway.

Blevins, R. D., 1977. Flow-induced Vibrations, New York: Van Nostrand Reinhold Company, 363.

Blevins, R. D. and Coughran, C. S., 2009. Experimental investigation of vortex-induced vibration in one and two dimensions with variable mass, damping, and Reynolds number, Journal of Fluids Engineering, Transactions of the ASME, 131(10): 101202.

Brika, D. and Laneville, A., 1993. Vortex-induced vibrations of a long flexible circular cylinder, J. Fluid Mech., 250, 481 508.

Chen, W. M., Zhang, L. W. and Li, M., 2010. Prediction of vortex-induced vibration of flexible riser using improved wake-oscilator model, Engineering Mechanics, 27(5): 240 246. (in Chinese)

Farshidianfar, A. and Zanganeh, H., 2010. A modified wake oscillator model for vortex-induced vibration of circular cylinders for a wide range of mass-damping ratio, Journal of Fluids and Structures, 26(3): 430 441.

Feng, C. C., 1968. The Measurement of Vortex-induced Effects on Flow past Stationary and Oscillating Circular and D-section Cylinders, M. Sc. Thesis, The university of British Columbia, Columbia.

Fredsoe, J., Sumer, B. M., Andersen, J., and Hansen, E. A., 1985. Transverse vibration of a cylinder very close to a plane wall, Proceedings of 4th International Symposium On Offshore mechanics and Arctic Engineering, ASME, Gopalkrishnan, 1, 601 609.

Govardhan, R. and Williamson, C. H. K., 2000. Modes of vortex formation and frequency response for a freely-vibrating cylinder, J. Fluid Mech., 420, 85 130.

Govardhan, R. and Williamson, C. H. K., 2004. Critical mass in vortex-induced vibration of a cylinder, European Journal of Mechanics B/Fluids, 23(1): 17 27.

Govardhan, R. and Williamson, C. H. K., 2006. Defining the 'modified Griffin plot' in vortex-induced vibration: revealing the effect of Reynolds number using controlled damping, J. Fluid Mech., 561, 147 180.

Huang, W. P., Cao, J., Zhang, E. Y. and Tang, S. Z., 2011. On vortex induced vibration in two-degree-of-freedoms of flexible cylinders, Chinese Journal of Theoretical and Applied Mechanics, 43(2): 436 440. (in Chinese)

Horowitz, M. and Williamson, C. H. K., 2010. Vortex-induced vibration of a rising and falling cylinder, J. Fluid Mech. 662, 352 383.

Jacobsen, V., Bryndum, M. B., Nielsen, R. and Fines, S., 1984. Cross-flow vibration of a pipe close to a rigid boundary, Journal of Energy Resources Technology, Transactions of ASME, 106, 451 457.

Jauvtis, N. and Williamson, C. H. K., 2003. Vortex-induced vibration of a cylinder with two degrees of freedom, Journal of Fluids and Structures, 17(7): 1035 1042.

Khalak, A. and Williamson, C. H. K., 1996. Dynamics of a hydroelastic cylinder with very low mass and damping, Journal of Fluids and Structures, 10(5): 455 472. 
Khalak, A. and Williamson, C. H. K., 1997a. Investigation of the relative effects of mass and damping in vortex-induced vibration of a circular cylinder, Journal of Wind Engineering and Industrial Aerodynamics, 69-71, 341 350.

Khalak, A. and Williamson, C. H. K., 1997b. Fluid forces and dynamics of a hydroelastic structure with very low mass and damping, Journal of Fluids and Structures, 11, 973 982.

Khalak, A. and Williamson, C. H. K., 1999. Motions, forces and mode transitions in vortex-induced vibrations at low mass-damping, Journal of Fluids and Structures, 13(7-8): 813 851.

King, R. and Jones, R., 1980. Flow-induced Vibrations of an Anchor Agitator, Practical Experiences with Flow-Induced Vibrations, Springer-Verlag, New York, 323 332.

Moe, G. and Wu, Z. J., 1990. The lift force on a cylinder vibrating in a current, Journal of Offshore Mechanics and Arctic Engineering, ASME, 112, 297 303.

Raven, P. W. J. and Stuart, R. J., 1985. Full-scale dynamic testing of submarine pipeline spans, OTC, Houston, Texas, 5005, 395 404.

Raghavan. K. and Bernitsas, M. M., 2011. Experimental investigation of Reynolds number effect on vortex induced vibration of rigid circular cylinder on elastic supports, Ocean Eng., 38(5-6): 719 731.

Sarpkaya, T., 1995. Hydrodynamic damping, flow-induced oscillations, and biharmonic response, Journal of Offshore Mechanics and Arctic Engineering, ASME, 117(4): 232 238.

Shen, Z. H., Liu, Y. B., Li, Q. P., Huang, Q. H. and Zhu, F. R., 2000. Experiments on interaction between current-induced vibration and scour of submarine pipelines on sandy bottom, China Ocean Eng., 14(4): 423 434.

Sumer, B. M., Mao, Y. and Fredsøe, J., 1988. Interaction between vibrating pipe and erodible bed, J. Waterw. Port Coast. Ocean Eng., 114(1): 81 92.

Tang, G. Q., Lv, L., Teng, B., Xie, B., Song. J. N., Zhang, J. Q. and Wu, H., 2011. Laboratory measurement of vortex-induced vibration of long flexible riser, The Ocean Engineering, 29(1): 18 25. (in Chinese)

Tsahalis, D. T. and Jones, W. T., 1981. Vortex-induced vibrations of a flexible cylinder near a plane boundary in steady flow, Proceedings of the 13th Annual Offshore Technology Conference, Houston, Tex., OTC Paper No. 3991, 367 372.

Tsahalis, D. T. ,1984. Vortex-induced vibrations of a flexible cylinder near a plane boundary exposed to steady and wave-induced currents, Journal of Energy Resources Technology, Transactions of ASME, 106(2): 206 213.

Wilson, J. F. and Caldwell, H. M., 1971. Force and stability measurements on models of submerged pipelines, Journal of Engineering for Industry, Transactions of the ASME, 93, 1290 1298.

Williamson, C. H. K. and Govardhan, R., 2004. Vortex-induced vibrations, Annual Review of Fluid Mechanics, 36, 413 455.

Williamson, C. H. K. and Govardhan, R., 2008. A brief review of recent results in vortex-induced vibrations, Journal of Wind Engineering and Industrial Aerodynamics, 96(6-7): 713 735.

Williamson, C. H. K. and Jauvtis, N., 2004. A high-amplitude 2T mode of vortex-induced vibration for a light body in xy motion, European Journal of Mechanics B/Fluids, 23(1): 107 114.

Yang, B., Gao, F. P. and Wu, Y. X., 2008a. Flow-induced vibrations of a cylinder with two degrees of freedom near rigid plane boundary, International Journal of Offshore and Polar Engineering, 18(4): 302 307.

Yang, B., Gao, F. P., Jeng, D. S. and Wu, Y. X., 2008b. Experimental study of vortex-induced vibrations of a pipeline near an erodible sandy seabed, Ocean Eng., 35(3-4): 301 309.

Yang, B., Gao, F. P., Wu, Y. X. and Li D. H., 2006. Experimental study on vortex-Induced vibrations of submarine pipeline near seabed boundary in ocean currents, China Ocean Eng., 20(1): 113 121. 